hep-th/0206125

IHES/P/02/43

ULB-TH-02/18

\title{
Einstein billiards and overextensions of finite-dimensional simple Lie algebras
}

\author{
Thibault Damour ${ }^{a}$, Sophie de Buyl ${ }^{b}$, Marc Henneaux $^{b, c}$ \\ and \\ Christiane Schomblond ${ }^{b}$ \\ ${ }^{a}$ Institut des Hautes Etudes Scientifiques, 35, Route de Chartres, F-91440 \\ Bures-sur-Yvette, France \\ ${ }^{b}$ Physique Théorique et Mathématique, Université Libre de Bruxelles, C.P. 231, \\ B-1050, Bruxelles, Belgium \\ ${ }^{c}$ Centro de Estudios Científicos, Casilla 1469, Valdivia, Chile
}

\begin{abstract}
In recent papers, it has been shown that (i) the dynamics of theories involving gravity can be described, in the vicinity of a spacelike singularity, as a billiard motion in a region of hyperbolic space bounded by hyperplanes; and (ii) that the relevant billiard has remarkable symmetry properties in the case of pure gravity in $d+1$ spacetime dimensions, or supergravity theories in 10 or 11 spacetime dimensions, for which it turns out to be the fundamental Weyl chamber of the Kac-Moody algebras $A E_{d}, E_{10}, B E_{10}$ or $D E_{10}$ (depending on the model). We analyse in this paper the billiards associated to other theories containing gravity, whose toroidal reduction to three dimensions involves coset models $G / H$ (with $G$ maximally non compact). We show that in each case, the billiard is the fundamental Weyl chamber of the (indefinite) Kac-Moody "overextension" (or "canonical Lorentzian extension") of the finite-dimensional Lie algebra that appears in the toroidal compactification to 3 spacetime dimensions. A remarkable feature of the billiard properties, however, is that they do not depend on the spacetime dimension in which the theory is analyzed and hence are rather robust, while the symmetry algebra that emerges in the toroidal dimensional reduction is dimension-dependent.
\end{abstract}




\section{Introduction}

\subsection{Einstein billiards}

This paper is devoted to a further investigation of the remarkable regularity properties of the billiards in hyperbolic space that control the asymptotic dynamics of theories involving gravity in the vicinity of a spacelike singularity.

Motivated by the work of Belinskii, Khalatnikov and Lifshitz [1], it was recently found that the dynamics of the Einstein-dilaton- $p$-form system in any number of spacetime dimensions can be described, near a spacelike singularity, as a billiard motion in a region of hyperbolic space [2, [3]. The dimension of the billiard, which will be called "Einstein billiard", and its precise shape depend on the theory at hand]. The emergence of hyperbolic geometry is related to the fact that the "supermetric" [6] in the space of spatial metrics has Lorentzian signature. [A detailed derivation of the billiards will be given in [7].]

It was further realized in [3] that the billiards associated (i) with the low energy bosonic sectors of 11-dimensional supergravity, or of types $I I_{A}$ and $I I_{B}$ supergravities in 10-dimensions, or with 10-dimensional type $I$ supergravities (ii) with or (iii) without a vector multiplet, could be identified with the fundamental Weyl chamber of the Kac-Moody algebra $E_{10}$ (case (i)), $B E_{10}$ (case (ii)) or $D E_{10}$ (case (iii)), which are all of indefinite type. These striking symmetry properties of the billiards relevant to the asymptotic dynamics hold also for pure gravity in any number of spacetime dimensions $D=d+1$, for which the billiard turns out to be the fundamental Weyl chamber of $A E_{d}[\mathbb{8}]$. The billiard dynamics is chaotic when the Kac-Moody algebra is hyperbolic [ $\left[\right.$, which is the case for $E_{10}, B E_{10}, D E_{10}$ and $A E_{d}$ with $d \leq 9$. Vacuum gravity is no longer chaotic in spacetime dimensions $\geq 11$, as observed previously in [9].

The purpose of this paper is to analyze in the light of these results other theories involving gravity, which have been found to be of interest in the past. These theories were introduced and classified by Breitenlohner, Maison and Gibbons [10]. The motivation of these authors in formulating such general classes of theories was to generalize the existence and uniqueness theorems known for black holes in Einstein-Maxwell theory. These theories, initially formulated in Ref. [10] as four-dimensional theories, have the property that,

\footnotetext{
${ }^{1}$ For four-dimensional vacuum gravity, one recovers the billiards described in [4, 5].
} 
when reduced to 3 spacetime dimensions, they describe three-dimensional gravity coupled to a coset model $G / H$, with a finite-dimensional symmetry group $G$. In many cases the initial four-dimensional theories can themselves be obtained from the dimensional reduction of higher-dimensional theories. On then says that they can be "oxidized" to higher dimensions. Their oxidation to higher dimensions has been systematically explored in [11] and we shall closely follow the formulation given in that paper. Some, but not all, of these theories are related to supergravity. As in [11], we concentrate on the cases where the numerator group $G$ of the three-dimensional coset is maximally non-compact ("normal" or "split" real form of the corresponding Lie algebra $\mathcal{G})$. We compute in each case the relevant billiard and find the remarkable result that the billiard is always the fundamental Weyl chamber of the "overextension" $\mathcal{G}^{\wedge \wedge}$ of the finite-dimensional Lie algebra $\mathcal{G}$ that appears in the toroidal compactification to three spacetime dimensions. We shall recall in Section 4 the definition of the overextension (or canonical Lorentzian extension) of a finite-dimensional Lie algebra $\mathcal{G}$, i.e. the canonical association to any $\mathcal{G}$ (of rank $r$ ) of a certain indefinite Kac-Moody algebra $\mathcal{G}^{\wedge \wedge}$ with rank $r^{\wedge \wedge}=r+2$. Let us only note beforehand that the Kac-Moody algebras $\mathcal{G}^{\wedge} \wedge$ are symmetrizable and that the metric in the Cartan subalgebra has Lorentzian signature, reflecting the signature of the supermetric for gravity.

Our main result, the sytematic appearance of the overextended symmetry algebra $\mathcal{G}^{\wedge \wedge}$, is a vast generalization of what was found to hold in the particular cases of eleven- and ten-dimensional supergravity theories [3] or pure gravity [8]. We note also that, if we view (as is explained in [12, 3, 7]) the cosmological billiards as a kind of "one-dimensional reduction" this result confirms the conjecture made long ago by Julia [13] concerning the systematic appearance of an "affine extension" $\mathcal{G}^{\wedge}$ in the reduction from 3 to 2 dimensions, to be followed by the appearance of an overextension $\mathcal{G}^{\wedge} \wedge$ in the further "reduction to one dimension" (in a sense which remained to be defined).

Contrary to what happens when considering (toroidal) dimensional reductions of a given higher-dimensional theory (for which the symmetry algebra explicitly depends on the dimension to which the model is reduced, i.e. on the height on the oxidation sequence), our billiard calculations can be performed in any number of dimensions, and always lead to the same result: namely the Weyl group of some Kac-Moody algebra. In practice, however, there are two preferred dimensions for doing the calculation: either the upper end of the oxidation sequence (i.e. the highest possible dimension; which corresponds 
to the most economical formulation of the theory), or its lower end (i.e. in 3 spacetime dimension; where the "initial" symmetry group $\mathcal{G}$ is present). In fact, both ways of doing the calculation have their respective merits. The highest-dimension calculation is technically the most straightforward. For this reason, we shall present it in detail in the main body of the paper in which we prove, by a case by case analysis, our main result. However, we shall present in our concluding section how a systematic reasoning done directly in the lowest dimension $(D=3)$ can elegantly detect the origin of the systematic appearance of an overextension.

\subsection{Organization of the paper and conventions}

Our paper is organized as follows. In the next section, we briefly recall how the billiards emerge from the dynamics in the vicinity of a spacelike singularity for the general system described by the Lagrangian

$$
\begin{aligned}
\mathcal{L}_{D}= & { }^{(D)} R \star \mathbb{1}-\sum_{\alpha} \star d \phi^{\alpha} \wedge d \phi^{\alpha} \\
& -\frac{1}{2} \sum_{p} e^{\lambda^{(p)}(\phi)} \star F^{(p+1)} \wedge F^{(p+1)}, \quad D \geq 3
\end{aligned}
$$

where $\lambda^{(p)}(\phi) \equiv \sum_{\alpha} \lambda_{\alpha}^{(p)} \phi^{\alpha}$, and where we have chosen units such that $16 \pi G=$ 1. We allow $N$ "dilatons" $\phi^{\alpha}(\alpha=1,2, \cdots, N)$ and normalize their kinetic terms with a weight 1 with respect to the Ricci scalar. The Einstein metric $g_{\mu \nu}$ has Lorentz signature $(-,+, \cdots,+)$ and is used to lower or raise the indices. Its determinant is ${ }^{(D)} g$. The integer $p \geq 0$ labels the various $p$-forms $A^{(p)}$ present in the theory, with field strengths $F^{(p+1)} \equiv d A^{(p)}$,

$$
F_{\mu_{1} \cdots \mu_{p+1}}^{(p+1)}=\partial_{\mu_{1}} A_{\mu_{2} \cdots \mu_{p+1}}^{(p)} \pm p \text { permutations }
$$

In fact, the field strength can be modified by additional coupling terms of Yang-Mills or Chapline-Manton type (e.g., $F_{C}^{(3)}=d C^{(2)}-C^{(0)} d B^{(2)}$ for two 2 -forms $C^{(2)}$ and $B^{(2)}$ and a 0 -form $C^{(0)}$, as it occurs in ten-dimensional type IIB supergravity). One can also add Chern-Simons terms. Although neither of these modifies the billiard analysis [2, 7], for the sake of completeness, we shall write them down explicitly below for the various theories under consideration (note that these terms are important for the symmetry group analysis of [11]). If there are several $p$-form gauge fields with the same form degree $p$, we use different letters $A^{(p)}, B^{(p)}, C^{(p)}, \ldots$ to distinguish them, as 
we just did. The real parameters $\lambda_{\alpha}^{(p)}$ measure the strength of the coupling to the "dilatons" $\phi^{\alpha}$. When $p=0$, we assume that $\lambda_{\alpha}^{(0)} \neq 0$ (for at least one $\alpha$ ), i.e., we assume that we have collected all the dilatons in the $\phi^{\alpha}$ 's. We shall also recall in section 2 the rules for computing the billiard walls as well as their angles and normalization; these rules have been given in [2, 3] and are explicitly written here in the case of many dilatons. We also verify that the billiard is invariant under toroidal dimensional reduction.

In section 3, we show that, if all the dilatons are absent, and if there are only proper $p$-forms, ? the billiard has always a finite volume. Hence, in that case, the system is always chaotic according to general results on hyperbolic billiards [14]. We also emphasize that although a billiard description of the asymptotic dynamics (as one approaches a spacelike singularity) always exists, it is only for special spacetime dimensions, menus of $p$-form and dilaton couplings that the billiard in question has notable regularity properties. In particular, the billiard associated with the gravity+3-form system is a Coxeter polyhedron only in 11 spacetime dimensions.

In section 4 , we recall the definition of overextensions of finite-dimensional Lie algebras [15, 13, 16]. We consider next the models associated with the classical groups (section 5) and the exceptional groups (section 6) and show that in each case, the billiard is the fundamental Weyl chamber of the overextension of the corresponding Lie algebra. Finally, we close our paper with some conclusions and with a streamlined argument explaining the origin of the systematic appearance of the overextensions.

\section{Recipes for constructing billiards}

\section{$2.1 \quad$ Billiard walls}

We recall the rules for constructing the billiards associated with the system (1.1) [2, 12, 7]. As one goes toward a spacelike singularity, the various spatial points effectively decouple [1]. At each spatial point, the degrees of freedom that carry the essential dynamics are the logarithms $\beta^{i} \equiv-\ln a_{i}$ of the scale factors $a_{i}$ along a set of (special) independent spatial directions $\left(d s^{2}=\right.$ $\left.-d t^{2}+\sum_{i} a_{i}^{2}(t, x)\left(\omega^{i}\right)^{2}\right)$ and the dilatons $\phi^{\alpha}$. [See [7] for the definition of

\footnotetext{
${ }^{2}$ We define "proper $p$-forms" as the $p$-forms with $0<p<D-2$. Indeed, $p=0$ corresponds to a scalar, $p=D-2$ is dual to a scalar and $p=D-1$ corresponds to the addition of a cosmological constant.
} 
the special vielbeins $\omega^{i}=e_{j}^{i}(t, x) d x^{j}$ leading to a simple billiard dynamics, as $t \rightarrow 0$, for the scale factors $a_{i}(t, x)$ after elimination of the off-diagonal components described by $e_{j}^{i}(t, x)$.] We denote collectively these variables by $\beta^{\mu}(\mu=1, \cdots, M)$ and shall loosely call all of them "scale factors". The total number of "scale factors" is therefore $M=d+N$, where $d$ is the spatial dimension and $N$ the number of dilatons. These variables are constrained (by the dynamics) to lie in a convex cone ("the wall cone") defined by a set of linear inequalities of the form

$$
w_{A}(\beta) \equiv w_{A \mu} \beta^{\mu} \geq 0 \quad A=1, \cdots Q .
$$

Here $Q$ is the number of walls, which depends on the system. The $w_{A \mu}$ are the "wall forms" and the hyperplanes $w_{A}(\beta)=0$ are the "walls". Note that the wall cone defined by the inequalities above does not depend on the normalization of the wall forms $w_{A \mu}$ (i.e. the same cone would be defined by considering wall forms $\lambda_{A} w_{A \mu}$ with $\lambda_{A}$ being any positive factor). However, the billiard dynamics that we consider is always characterized by a preferred set of wall forms, with a well-defined normalization. In fact, as shown in [3, [] our asymptotic dynamics is richer than a pure billiard dynamics with infinitely sharp walls. It is a (generalized) Toda dynamics, where each wall is a "Toda wall", i.e. corresponds to an exponential term $c_{A}^{2} \exp \left(-2 w_{A}(\beta)\right)$ in the Hamiltonian. The exponent appearing in these potential terms uniquely define our normalization for the wall forms.

Between two collisions against the walls, the scale factors move along a null straight line of the $M$-dimensional (where we recall that $M=d+N$ ) flat metric

$$
d S^{2}=G_{\mu \nu} \beta^{\mu} \beta^{\nu}=\sum_{i}\left(d \beta^{i}\right)^{2}-\left(\sum_{i} d \beta^{i}\right)^{2}+\sum_{\alpha}\left(d \phi^{\alpha}\right)^{2}
$$

This metric has Lorentzian signature $(-,+,+, \cdots,+)$. The inverse metric reads

$$
G^{\mu \nu} \partial_{\mu} f \partial_{\nu} f=\sum_{i}\left(\partial_{i} f\right)^{2}-\frac{1}{d-1}\left(\sum_{i} \partial_{i} f\right)^{2}+\sum_{\alpha}\left(\partial_{\alpha} f\right)^{2}
$$

for any function $f\left(\beta^{i}, \phi^{\alpha}\right)$. The future light cone of the metric is defined by $G_{\mu \nu} \beta^{\mu} \beta^{\nu}<0$ and $\sum_{i} \beta^{i}>0$.

The list of the walls that must be a priori considered is as follows:

1. Symmetry walls [3, 7]. These arise from the off-diagonal components of the metric and are cleanly derived using the Iwasawa decomposition 
of the spatial metric [7]. They read explicitly:

$$
w_{i j}^{S}(\beta)=\beta^{j}-\beta^{i}, \quad i<j
$$

2. Gravitational (or curvature) walls [2, 9]. These arise from the spatial curvature and are given by

$$
w_{i ; j k}^{G}(\beta)=2 \beta^{i}+\sum_{l \neq i, j, k} \beta^{\ell}, \quad i \neq j, i \neq k, j \neq k
$$

These walls are absent for $D=3$, i.e., there is no gravitational wall to be considered in that dimension. [There are, in all dimensions, subdominant gravitational walls of the form $w_{i}^{G}(\beta)=\sum_{\ell \neq i} \beta^{\ell}$. These walls are lightlike [7] and do not affect the asymptotic dynamics of the scale factors; this is why they have not been listed. Note that we assume at least one matter field when $D=3$ (which can be assumed to be a scalar field by dualization), since otherwise, there is no local degree of freedom and the only interesting dynamics is in the global degrees of freedom, not discussed here.]

3. Electric walls [2]. These arise from the electric energy density. For a $p$-form, the walls read

$$
w_{i_{1} \cdots i_{p}}^{E(p)}(\beta)=\beta^{i_{1}}+\cdots+\beta^{i_{p}}+\frac{1}{2} \sum_{\alpha} \lambda_{\alpha}^{(p)} \phi^{\alpha}, \quad i_{1}<\cdots<i_{p}
$$

4. Magnetic walls [2]. These arise from the magnetic energy density. For a $p$-form, they read

$$
w_{i_{1} \cdots i_{d-p-1}}^{M(p)}(\beta)=\beta^{i_{1}}+\cdots+\beta^{i_{d-p-1}}-\frac{1}{2} \sum_{\alpha} \lambda_{\alpha}^{(p)} \phi^{\alpha}, i_{1}<\cdots<i_{d-p-1}
$$

Not all these walls are relevant since the inequalities $w_{i j}^{S} \geq 0, w_{i ; j k}^{A} \geq 0$, $w_{i_{1} \cdots i_{p}}^{E} \geq 0$ and $w_{i_{1} \cdots i_{d-p-1}}^{M} \geq 0$ follow from the simpler subset

$$
\beta^{1} \leq \beta^{2} \cdots \leq \beta^{d}, \quad w_{1 ; 23}^{G} \geq 0, \quad w_{1 \cdots p}^{E} \geq 0, \quad w_{1 \cdots d-p-1}^{M} \geq 0
$$

This subset might still be redundant; e.g., in the presence of a proper $p$-form (i.e. with $0<p<D-2$ ), the gravitational wall forms can be written as sums of one electric and one magnetic wall form. So, once the list of all walls 
(2.8) has been written down for a given theory, the first task is to determine which among these walls are the "dominant" ones, i.e. the minimal set of walls which suffice to define the billiard.

It is easy to check that all the walls are timelike, i.e., that their normals are spacelike, $w_{A} \cdot w_{A}>0$ [2, 3]. In fact, the wall cone contains the future pointing timelike vector $\beta^{1}=\beta^{2}=\cdots=\beta^{d}=\alpha>0, \phi^{1}=\phi^{2}=\cdots=\phi^{N}=0$. The wall cone has therefore a non trivial intersection with the future light cone. Two cases can arise [8]

1. The wall cone is entirely contained in the light cone; in this case, the point representing the system in the space of the scale factors ("billiard ball") never stops hitting the walls.

2. The wall cone is not entirely contained in the light cone and contains thus some spacelike straight lines through the origin; in this case, the billiard ball will generically make a finite number of collisions with the walls and then go on freely forever, the directions of escape being parallel to the lightlike straight lines through the origin contained in the wall cone.

One can project the dynamics on the upper sheet of the unit hyperboloid $G_{\mu \nu} \beta^{\mu} \beta^{\nu}=-1$ [3]. This hyperboloid can be identified with the hyperbolic space $\mathcal{H}_{d+N-1}$. The walls define hyperplanes in $\mathcal{H}_{d+N-1}$. The interior region bounded by these hyperplanes is the billiard. The billiard is therefore the radial projection of the wall cone onto the unit hyperboloid. In case 1, the billiard has finite volume; in case 2, it has infinite volume.

\subsection{Dimensional reduction - Dualization}

The billiard is defined by the action (1.1) and by nothing else. One of its crucial properties is that it is invariant under toroidal compactification down to any spacetime dimension $\geq 3$ below the original spacetime dimension. The walls simply change name, but the billiard remains the same. Indeed, consider toroidal compactification of just one dimension (the general case is obtained by iteration) of (1.1) with only one $p$-form. The $D$-dimensional metric is related to the $(D-1)$-dimensional (Einstein) metric $\hat{g}_{\mu \nu}$, the KaluzaKlein $(\mathrm{KK})$ vector $\hat{\mathcal{A}}_{\mu}$ and the additional dilaton $\hat{\varphi}$ through the formulas

$$
g_{11}=e^{-2(d-2) \gamma \hat{\varphi}},
$$




$$
\begin{aligned}
& g_{1 \mu}=e^{-2(d-2) \gamma \hat{\varphi}} \hat{\mathcal{A}}_{\mu}, \quad \mu=0,2,3, \cdots, d \\
& g_{\mu \nu}=e^{2 \gamma \hat{\varphi}} \hat{g}_{\mu \nu}+e^{-2(d-2) \gamma \hat{\varphi}} \hat{\mathcal{A}}_{\mu} \hat{\mathcal{A}}_{\nu}
\end{aligned}
$$

where $\gamma=1 / \sqrt{(D-2)(D-3)}$. The $(D-1)$-form of the action reads

$$
\begin{aligned}
& \mathcal{L}_{D-1}={ }^{(D-1)} \hat{R} \star \mathbb{1}-\star d \hat{\varphi} \wedge d \hat{\varphi}-\frac{1}{2} e^{-2(D-2) \gamma \hat{\varphi}} \star \hat{\mathcal{F}} \wedge \hat{\mathcal{F}}-\sum_{\alpha} \star d \hat{\phi}^{\alpha} \wedge d \hat{\phi}^{\alpha} \\
& -\frac{1}{2} e^{\lambda_{\alpha}^{(p)} \hat{\phi}^{\alpha}-2 p \gamma \hat{\varphi}} \star \hat{F}^{(p+1)} \wedge \hat{F}^{(p+1)}-\frac{1}{2} e^{\lambda_{\alpha}^{(p)} \hat{\phi}^{\alpha}+2(D-2-p) \gamma \hat{\varphi}} \star \hat{F}^{(p)} \wedge \hat{F}^{(p)}(2.12)
\end{aligned}
$$

where $\hat{\mathcal{F}}$ is the field strength of the K-K vector potential and where $\hat{F}^{(p+1)}$ and $\hat{F}^{(p)}$ denote the field strengths of the $p$-form and the $(p-1)$-form originating from the $p$-form in $D$ dimensions.

Let $\hat{\beta}^{a}(a=2, \cdots, d)$ be the scale factors in $D-1$ spacetime dimensions. The relationship between $\hat{\varphi}, \hat{\beta}^{a}$ and the original scale factors $\beta^{i}(i=1, a)$ in $D$ spacetime dimensions is

$$
\beta^{a}=\hat{\beta}^{a}-\gamma \hat{\varphi}, \quad \beta^{1}=(d-2) \gamma \hat{\varphi} .
$$

Given this relationship, one easily verifies by mere substitution that

- The $d(d-1) / 2$ symmetry walls (2.4) give rise to $(d-1)(d-2) / 2$ symmetry walls in $(D-1)$ dimensions

$$
\hat{w}_{a b}^{S}=\hat{\beta}^{b}-\hat{\beta}^{a}, a<b
$$

and to the $(d-1)$ electric walls of the K-K 1-form:

$$
\hat{w}_{a}^{E, K K}=\hat{\beta}^{a}-(d-1) \gamma \hat{\varphi} .
$$

- The $d ! / p !(d-p)$ ! electric walls (2.6) give rise to the $(d-1) ! / p !(d-1-p)$ ! electric walls of the $p$-form:

$$
\hat{w}_{a_{1} \cdots a_{p}}^{E, p}=\hat{\beta}^{a_{1}}+\cdots+\hat{\beta}^{a_{p}}+\frac{\lambda_{\alpha}^{(p)}}{2} \hat{\phi}^{\alpha}-p \gamma \hat{\varphi}
$$

and to the $(d-1) ! /(p-1) !(d-p)$ ! electric walls of the $(p-1)$-form:

$$
\hat{w}_{a_{1} \cdots a_{p-1}}^{E, p-1}=\hat{\beta}^{a_{1}}+\cdots+\hat{\beta}^{a_{p-1}}+\frac{\lambda_{\alpha}^{(p)}}{2} \hat{\phi}^{\alpha}+(d-1-p) \gamma \hat{\varphi}
$$


- The $d ! /(p+1) !(d-1-p)$ ! magnetic walls (2.7) give rise to the $(d-$ $1) ! /(p+1) !(d-2-p)$ ! magnetic walls of the $p$-form:

$$
\hat{w}_{a_{1} \cdots a_{d-2-p}}^{M, p}=\hat{\beta}^{a_{1}}+\cdots+\hat{\beta}^{a_{d-2-p}}-\frac{\lambda_{\alpha}^{(p)}}{2} \hat{\phi}^{\alpha}+p \gamma \hat{\varphi}
$$

and to the $(d-1) ! / p !(d-1-p) !$ magnetic walls of the $(p-1)$-form:

$$
\hat{w}_{a_{1} \cdots a_{d-1-p}}^{M, p-1}=\hat{\beta}^{a_{1}}+\cdots+\hat{\beta}^{a_{d-1-p}}-\frac{\lambda_{\alpha}^{(p)}}{2} \hat{\phi}^{\alpha}-(d-1-p) \gamma \hat{\varphi}
$$

The situation with the gravitational walls is slightly more subtle. Indeed, while it is easy to see that the curvature walls (2.5) with $(i, j, k)=(a, b, c)$ are just the curvature walls in $(D-1)$ dimensions:

$$
w_{a ; b c}^{G}=\hat{w}_{a ; b c}^{G}=2 \hat{\beta}^{a}+\sum_{g \neq a, b, c} \hat{\beta}^{g}, \quad a \neq b, a \neq c, b \neq c
$$

and that the curvature walls (2.5) with $(i, j, k)=(1, a, b)$ are just the $(d-$ 1) $(d-2) / 2$ magnetic walls of the K-K 1 -form (with $\left\{a_{1}, \ldots, a_{d-3}\right\}$ in the complementary subset to $\{a, b\})$ :

$$
\hat{w}_{a_{1} \ldots a_{d-3}}^{M, K K}=\hat{\beta}^{a_{1}}+\cdots+\hat{\beta}^{a_{d-3}}+(d-1) \gamma \hat{\varphi},
$$

one finds that the original gravitational walls $w_{a ; 1 b}^{G}$ are absent in the $(D-1)$ dimensional theory. This is because the corresponding walls are multiplied, in the $D$-dimensional theory, by the (square of the) structure constants $C^{a}{ }_{1 b}$ [7], which are zero under the dimensional reduction assumption that the fields do not depend on the coordinate $x^{1}$. The fact that the walls $w_{a ; 1 b}^{G}$ are absent in the $(D-1)$-dimensional theory is, however, not a problem because these walls are always subdominant: the corresponding wall forms can be expressed as linear combinations with positive (integer) coefficients of the other gravitational wall forms and the symmetry wall forms.

Another important - and rather obvious - property of the set of walls is that it is invariant under electric-magnetic duality transformations, in which one replaces a $p$-form by a $(D-p-2)$-form such that the curvatures are dual to one another. This changes the sign of the dilaton coupling and exchanges electric and magnetic walls. Note that the squared norm of the electric and magnetic walls associated with the same $p$-form are equal. 


\subsection{Coxeter polyhedra - Kac-Moody billiards}

A convex polyhedron in $\mathcal{H}_{d+N-1}$ is a finite intersection of half spaces of $\mathcal{H}_{d+N-1}$ with a non-empty interior; our billiards are thus convex polyhedra in $\mathcal{H}_{d+N-1}$. A Coxeter polyhedron is a polyhedron such that the dihedral angles between adjacent faces are integer submultiples of $\pi$ (i.e., of the form $\pi / k$ where $k$ is an integer $\geq 2$ ). For a Coxeter polyhedron, the group generated by the reflections in the faces is a discrete subgroup of the isometry group of $\mathcal{H}_{d+N-1}$ (Poincaré theorem). It is in fact a Coxeter group admitting the following presentation (see 17 for details on discrete reflection groups in hyperbolic spaces): let $s_{i}$ be the reflection in the face $i$ and let $\pi / m_{i j}$ be the dihedral angle between the faces $i$ and $j$, with $m_{i j}=1$ if $i=j$, and $m_{i j}=\infty$ if $i$ and $j$ are not adjacent [The hyperplanes of non-adjacent faces of a Coxeter polyhedron do not intersect [17]]. Then, the presentation of the group generated by the $s_{i}$ 's is

$$
s_{i}^{2}=1, \quad\left(s_{i} s_{j}\right)^{m_{i j}}=1
$$

Although the billiards defined by the action (1.1) are polyhedra, they fail, in general, to be Coxeter polyhedra. This is illustrated in section 3 below. However, for the "interesting systems" considered in this paper, the menu of $p$-forms, the dilaton couplings and the spacetime dimension conspire to yield polyhedra with faces that meet precisely with angles that are submultiples of $\pi$. One has actually even more, namely, for all the systems considered below

- The billiard is a simplex, i.e., has exactly $d+N$ faces. We denote from now on by $w_{i}=0(i=-1,0 \cdots, d+N-2)$ the corresponding relevant faces.

- The dominant wall forms $w_{i}$ have scalar products such that the matrix

$$
A_{i j}=2 \frac{\left(w_{i} \mid w_{j}\right)}{\left(w_{i} \mid w_{i}\right)} \quad \text { where } \quad\left(w_{i} \mid w_{j}\right) \equiv G^{\mu \nu} w_{i \mu} w_{j \nu}
$$

is a generalized Cartan matrix, i.e., has non-positive integer off-diagonal components [16] (the other properties are automatic consequences from the definition: $A_{i i}=2$ and $\left.A_{i j}=0 \Rightarrow A_{j i}=0\right)$.

We shall say that a billiard with these special properties is a (Lorentzian) Kac-Moody billiard. By contrast with the definition of a Coxeter billiard, 
i.e. motion within a Coxeter polyhedron, which depended only on dihedral angles, the definition of a Kac-Moody billiard depends, through the definition (2.23) of the Cartan matrix, on the normalization of the wall forms. But, as we said, our Toda-like billiard comes equipped with specially-normalized wall forms. For a Kac-Moody billiard, the only possible values of $m_{i j}$ are 1 $(i=j), 2,3,4,6$ and $\infty$.

When $A_{i j}$ is a generalized Cartan matrix, it defines a Kac-Moody algebra of rank $d+N$, symmetrizable, of indefinite type, with a scalar product of Lorentzian signature (the scalar product is just (2.2)). The Kac-Moody billiard defined by the inequalities $w_{i}(\beta) \geq 0$ can then be identified with the fundamental Weyl chamber of the Kac-Moody algebra (more precisely, is the radial projection on the unit hyperboloid of the intersection of the fundamental Weyl chamber with the light cone). Among Lorentzian Kac-Moody algebras, those whose Dynkin diagram is such that the removal of any node yields the Dynkin diagram of a Kac-Moody algebra of finite or affine type are called "hyperbolic". Hyperbolic Kac-Moody algebras are important in the present context: Kac-Moody billiards have finite volume (and hence are chaotic) if and only if the underlying algebra is hyperbolic [8].

\section{Some general results}

As expected from the discussion of the previous section, Kac-Moody billiards, being quite special, must be rather exceptional. The purpose of this section is to show explicitly that this is indeed the case. This is rather clear when there are dilatons, because the angles between the faces of the billiards depend on the dilaton couplings, which are continuous parameters. By continuously changing these parameters, one continuously change the angles, which are therefore integer submultiples of $\pi$ only for special values of the dilaton couplings.

The fact that Kac-Moody billiards are rare is also true in the absence of dilatons. To establish this fact, we first prove the following

Theorem 3.1 : Assume that there is no dilaton and that there is at least one proper $p$-form $(0<p<d-1)$. Then, the billiard has finite volume (and is thus chaotic) in any number of spacetime dimensions $(\geq 3)$.

To demonstrate this theorem, we show that the billiard for the system gravity + a single proper $p$-form $(0<p<d-1)$ has finite volume. Since 
the billiard for a collection of $p$-forms is the intersection of the individual billiards with a single $p$-form involved, the result will follow. Without loss of generality, we can assume $2 p<d$ because the set of walls is invariant under electric-magnetic duality. The walls are explicitly

$$
\begin{aligned}
& \beta^{1} \leq \beta^{2} \leq \cdots \leq \beta^{d} \quad \text { (symmetry walls) } \\
& \beta^{1}+\cdots+\beta^{p} \geq 0 \quad(\text { electric wall) } \\
& \beta^{1}+\cdots+\beta^{d-p-1} \geq 0 \quad \text { (magnetic wall) }
\end{aligned}
$$

Since $d-p-1 \geq p$, the relevant walls are the symmetry and electric walls. The billiard is defined by

$$
\begin{aligned}
& \beta^{1} \leq \beta^{2} \leq \cdots \leq \beta^{d} \quad \text { (symmetry walls) } \\
& \beta^{1}+\cdots+\beta^{p} \geq 0 \quad \text { (electric wall) }
\end{aligned}
$$

and is a simplex. Our claim is that the billiard has finite volume, or, equivalently, that the wall cone (3.4), (3.5) is contained within the light cone. That is, all the $d$ intersection edges of any subset of $d-1$ faces are timelike or null. The edge opposite to the electric wall is $\left(\beta^{1}, \beta^{2}, \cdots, \beta^{d}\right)=(\alpha, \alpha, \cdots, \alpha)$ with $\alpha>0$ and is timelike since $\alpha^{2} d(1-d)<0$. The other edges are obtained by dropping one of the symmetry equalities. Vectors along these edges can be taken to be

$$
(-(p-k),-(p-k), \cdots,-(p-k), k, k, \cdots, k), \quad k=1,2, \cdots, p
$$

(first $k$ components equal to $-(p-k)$, last $d-k$ components equal to $k$ ) and

$$
(0,0, \cdots, 0,1, \cdots, 1) \quad k=p+1, \cdots, d-1
$$

(first $k$ components equal to 0 , last $d-k$ components equal to 1 ). The edges (3.7) are easily seen to have squared norm equal to $(d-k)(1+k-d)$ and are timelike $(k<d-1)$ or null $(k=d-1)$. The edges (3.6) have squared norm equal to

$$
k\left[-k\left(p^{2}+2(1-d) p+d(d-1)\right)+p^{2}\right]
$$

The coefficient of $k$ in the bracket is always strictly negative, which means that the expression in the bracket is a monotonously decreasing function of $k$. Accordingly, if we show that (3.8) is negative for $k=1$, it will be automatically negative for $k>1$. Now, for $k=1$, the bracket in (3.8) reads

$$
-\left(p^{2}+2(1-d) p+d(d-1)\right)+p^{2}
$$


Setting $d=2 p+s$ with $s>0$, this becomes

$$
-s(s+2 p-1)
$$

which is strictly negative. Hence, all the edges are timelike or null and the billiard has finite volume. [We have also learned that there is only one lightlike edge, and hence, only one vertex at infinity for a billiard with a single $p$-form and no dilaton.] $\square$.

Note that the we need in fact only $d \geq 2 p$ instead of the strict inequality $d>2 p$ to achieve this result, since when $d=2 p$, there is just one additional edge on the light cone, but this still gives a finite-volume billiard. [However, when $d=2 p$, the magnetic wall dominates.] This shows in particular that the electric walls are sufficient to drive the chaos when $d \geq 2 p$; otherwise chaos is magnetically-driven. The particular cases $p=1,2,3$ were investigated previously in [12].

A direct application of the above theorem is:

Theorem 3.2 : Consider gravity coupled to a collection of proper $p$-forms, with no dilaton $(0<p<d-1$ for each $p$-form). If the spacetime dimension $D$ is strictly greater than $11, D>11$, the corresponding billiard cannot be a Kac-Moody billiard.

Indeed, if it were, the corresponding Kac-Moody algebra should be hyperbolic since the system is chaotic. But there is no hyperbolic Kac-Moody algebra of rank strictly greater than 10 [16]. $\square$.

We now consider the particular case of gravity coupled to a 3-form and investigate when the associated billiard is a Coxeter polyhedron.

Theorem 3.3 : Consider the Einstein-3-form system in spacetime dimension $D \geq 6$. The billiard associated with this system is a Coxeter polyhedron if and only if $D=11$.

Note the restriction to $D \geq 6$ which corresponds to the case where the 3 -form is proper: $p=3<D-2$. The billiard for this system is a simplex bounded by the $d-1$ symmetry walls and the magnetic wall

$$
\beta^{1} \geq 0
$$

for $D=6$, the magnetic wall

$$
\beta^{1}+\beta^{2} \geq 0
$$


for $D=7$ and the electric wall

$$
\beta^{1}+\beta^{2}+\beta^{3} \geq 0
$$

for $D \geq 8$. The magnetic wall (3.9) is orthogonal to all the symmetry walls except $\beta^{2}-\beta^{1}=0$, with which it makes an angle equal to $\pi / 5.104301$, which is not an integer submultiple of $\pi$. The magnetic wall (3.10) is orthogonal to all the symmetry walls except $\beta^{3}-\beta^{2}=0$, with which it makes an angle equal to $\pi / 3.614672$, which is not an integer submultiple of $\pi$. Finally, the electric wall (3.11) is orthogonal to all the symmetry walls except $\beta^{4}-\beta^{3}=0$, with which it makes an angle $\hat{\alpha}$ given by

$$
\cos \hat{\alpha}=\frac{1}{\sqrt{6}} \sqrt{1+\frac{3}{d-4}} .
$$

This angle is an integer submultiple of $\pi$ only for $D=11$, in which case it is equal to $\pi / 3$ and the billiard is the Kac-Moody billiard of $E_{10}$ [3].

From this point of view, $D=11$ is thus quite special for the gravity-3form system, irrespective of any supersymmetry consideration. If one did not know about supergravity, but had some independent reason for considering a 3 -form, one could discover from this independent line of insight the peculiar rôle of $D=11$.

Note: One checks along similar lines that besides the case just considered (and its dual $D=11, p=6$ formulation), the only gravity $+p$-form systems (with a single $p$-form) that lead to Coxeter polyhedra are: (i) gravity + a 1form in $D=4$ and $D=5$ spacetime dimensions, discussed in [8]; (ii) gravity + a 2 -form in $D=5$ and $D=6$ spacetime dimensions (the first case is dual to the Einstein-Maxwell theory in 5 dimensions and yield thus $G_{2}^{\wedge \wedge}[\mathbb{8}$; the second case is easily verified to yield $B_{3}^{\wedge \wedge}$ and is the oxidation endpoint of the $B_{3}$-sequence [11]); and (iii) gravity + a 4-form in 10 dimensions, which yields $E_{7}^{\wedge}$ and is one of the natural oxidation endpoints of the $E_{7}$-sequence [11] (see below for detailed computations).

\section{Overextension of finite-dimensional Lie al- gebras}

Following [15, 13, 16], we define the overextended algebra $\mathcal{G}^{\wedge \wedge}$ of a finitedimensional Lie algebra $\mathcal{G}$ through its roots and generalized Cartan matrix. 
The general construction goes as follows. Let $R$ denote the root space of a finite-dimensional Lie algebra $\mathcal{G}$ of rank $d$, and Cartan matrix $A$. Let $\left\{\alpha_{i}, i=1, \ldots, d\right\}$ be a (particular) set of simple roots, so that

$$
A_{i j}=2 \frac{\left(\alpha_{i} \mid \alpha_{j}\right)}{\left(\alpha_{i} \mid \alpha_{i}\right)}
$$

where (.|.) denotes the standard invariant form on $R$, and let $\theta=\sum_{i} n_{i} \alpha_{i}$ be the corresponding highest root (with the $n_{i}$ being some uniquely defined positive integers). Let us normalize the standard invariant form on $R$ by the condition $(\theta \mid \theta)=2$. As a finite-dimensional Lie algebra has roots of at most two different lengths, this normalization means that all the "long roots" have a squared length 2 .

Let $V$ be the 2-dimensional hyperbolic space with basis $u_{1}, u_{2}$ and bilinear form of signature $(-,+)$

$$
\left(u_{1} \mid u_{2}\right)=1, \quad\left(u_{1} \mid u_{1}\right)=\left(u_{2} \mid u_{2}\right)=0 .
$$

Then, we define the canonical Lorentzian extension of $R$ as the orthogonal direct sum $V \oplus R$. It is equipped with an inner product of Lorentzian signature $(-,+,+, \ldots+)$. [ Note that Kac [16] calls it the canonical hyperbolic extension of $R$, but because the corresponding Kac-Moody algebra need not be hyperbolic, we prefer to use the more neutral adjective "Lorentzian".] Defining the following basis of $V \oplus R$

$$
\begin{aligned}
& \alpha_{-1}=u_{1}+u_{2} \\
& \alpha_{0}=-u_{2}-\theta \\
& \alpha_{i} \quad(i=1, \ldots, d)
\end{aligned}
$$

one can form the $(d+2) \times(d+2)$ matrix

$$
A_{\mu \nu}^{\wedge \wedge}=2 \frac{\left(\alpha_{\mu} \mid \alpha_{\nu}\right)}{\left(\alpha_{\mu} \mid \alpha_{\mu}\right)} \quad(\mu, \nu=-1,0, \ldots, d) .
$$

This matrix is easily verified to be a generalized Cartan matrix. One then defines the "overextension" or "canonical Lorentzian extension" of the original finite-dimensional Lie algebra $\mathcal{G}$ as the Kac-Moody algebra built on the generalized Cartan matrix $A^{\wedge \wedge}$. We shall denote this (infinite-dimensional) overextended Lie algebra as $\mathcal{G}^{\wedge \wedge}$. It has rank $d+2$. 
One can also consider the Kac-Moody algebra generated by the "onceextended" set of $(d+1)$ simple roots $\left(\alpha_{0}, \alpha_{i}\right)$. The corresponding $(d+1) \times$ $(d+1)$ matrix $A^{\wedge}$ is also a generalized Cartan matrix, albeit a degenerate one ( $\left.\operatorname{det} A^{\wedge}=0\right)$. This once-extended Kac-Moody algebra, denoted $\mathcal{G}^{\wedge}$, is called the "untwisted affine extension" of $\mathcal{G}$. The root $\alpha_{0}$ is called the "affine root", while $\alpha_{-1}$ is called the "overextended" root. It is orthogonal to all others except $\alpha_{0}$ :

$$
\left(\alpha_{-1} \mid \alpha_{0}\right)=-1, \quad\left(\alpha_{-1} \mid \alpha_{i}\right)=0
$$

The Dynkin diagram of $\mathcal{G}^{\wedge} \wedge$ is obtained from the corresponding diagram of the affine extension $\mathcal{G}^{\wedge}$ by adding a vertex $\alpha_{-1}$ joined with the affine vertex $\alpha_{0}$ by a simple link.

\section{Models associated with classical groups}

Let us consider in turn the different $G / H$ models introduced in 10 and further studied in [11]. They are all naturally labelled by the numerator groups $G$, or equivalently (knowing that we consider only the maximally noncompact real forms of the group) by the corresponding (finite-dimensional) Lie algebras: successively $A_{n}, B_{n}, C_{n}$ and $D_{n}$. We shall consider the models based on exceptional groups in the next section.

\subsection{The $A_{n}$ sequence}

The $A_{n}$ sequence is by definition such that the symmetry group in 3 spacetime dimensions is $S L(n+1, R)$. The maximal oxidation point of the $A_{n}$ sequence is pure gravity in $D=n+3$ spacetime dimension [11]. The Lagrangian is thus

$$
\mathcal{L}_{D}=R \star \mathbb{1}, \quad D=n+3
$$

There are only symmetry and gravitational walls. The billiard is defined by the conditions

$$
\beta^{1} \leq \beta^{2} \cdots \leq \beta^{d}, 2 \beta^{1}+\beta^{2}+\cdots+\beta^{d-2} \geq 0, \quad d=n+2
$$

and is a simplex. The computation of the Cartan matrix has been done [8], where it was found that $A_{i j}$ is just the generalized Cartan matrix of the 
overextension $A_{n}^{\wedge \wedge}$ of the Lie algebra $A_{n} \cdot\left[A_{n}^{\wedge \wedge}\right.$ is also denoted $\left.A E_{(n+2)}\right]$. For $d>3$, it reads

$$
A=\left(\begin{array}{cccccccc}
2 & -1 & 0 & 0 & \cdots & 0 & 0 & 0 \\
-1 & 2 & -1 & 0 & \cdots & 0 & 0 & -1 \\
0 & -1 & 2 & -1 & \cdots & 0 & 0 & 0 \\
\vdots & \vdots & \vdots & \vdots & \cdots & \vdots & \vdots & \vdots \\
0 & 0 & 0 & 0 & \cdots & 2 & -1 & 0 \\
0 & 0 & 0 & 0 & \cdots & -1 & 2 & -1 \\
0 & -1 & 0 & 0 & \cdots & 0 & -1 & 2
\end{array}\right)
$$

As explained above, the computation could have been done in any dimension between 3 and $n+3$ with identical conclusions. The corresponding Dynkin diagram is given in figure 1. The algebra is hyperbolic for $n<8$. The extension $A E_{9} \equiv A_{7}^{\wedge}$ is the last hyperbolic algebra in the family; $A E_{10} \equiv A_{8}^{\wedge \wedge}$ is not hyperbolic.

\subsection{The $B_{n}$ sequence}

The oxidation endpoint is $D=n+2$ [11]. The theory comprises the metric, a dilaton, a 2 -form, $B$, and a 1 -form, $A$. The Lagrangian reads

$$
\mathcal{L}_{D}=R \star \mathbb{1}-\star d \phi \wedge d \phi-\frac{1}{2} e^{a \sqrt{2} \phi} \star G \wedge G-\frac{1}{2} e^{a \frac{\sqrt{2}}{2} \phi} \star F \wedge F,
$$

where $a^{2}=8 / n$ and

$$
G=d B+\frac{1}{2} A \wedge d A, \quad F=d A
$$

In addition to the symmetry and gravitational walls, one has electric and magnetic walls for the 2-form,

$$
w_{i j}^{E, B}=\beta^{i}+\beta^{j}+a \frac{\sqrt{2}}{2} \phi, \quad w_{i_{1} \ldots i_{n-2}}^{M, B}=\beta^{i_{1}}+\cdots+\beta^{i_{n-2}}-a \frac{\sqrt{2}}{2} \phi
$$

as well as electric and magnetic walls for the 1-form,

$$
w_{i}^{E, A}=\beta^{i}+a \frac{\sqrt{2}}{4} \phi, \quad w_{i_{1} \cdots i_{n-1}}^{M, A}=\beta^{i_{1}}+\cdots+\beta^{i_{n-1}}-a \frac{\sqrt{2}}{4} \phi .
$$


It is easy to see that the billiard can be completely defined by the following $n+2$ independent walls

$$
\begin{aligned}
& w_{-1}=\beta^{n+1}-\beta^{n} \\
& w_{0}=\beta^{n}-\beta^{n-1} \\
& w_{1}=\beta^{1}+\ldots+\beta^{n-2}-a \frac{\sqrt{2}}{2} \phi \\
& w_{2}=\beta^{n-1}-\beta^{n-2} \\
& w_{3}=\beta^{n-2}-\beta^{n-3} \\
& \ldots \\
& w_{n-1}=\beta^{2}-\beta^{1} \\
& w_{n}=\beta^{1}+a \frac{\sqrt{2}}{4} \phi .
\end{aligned}
$$

A straighforward computation yields the following generalized Cartan matrix

$$
A=\left(\begin{array}{ccccccccc}
2 & -1 & 0 & 0 & \cdots & 0 & 0 & 0 & 0 \\
-1 & 2 & 0 & -1 & \cdots & 0 & 0 & 0 & 0 \\
0 & 0 & 2 & -1 & \cdots & 0 & 0 & 0 & 0 \\
0 & -1 & -1 & 2 & \cdots & 0 & 0 & 0 & 0 \\
\vdots & \vdots & \vdots & \vdots & \ddots & \vdots & \vdots & \vdots & \vdots \\
0 & 0 & 0 & -1 & \cdots & 2 & -1 & 0 & 0 \\
0 & 0 & 0 & 0 & \cdots & -1 & 2 & -1 & 0 \\
0 & 0 & 0 & 0 & \cdots & 0 & -1 & 2 & -1 \\
0 & 0 & 0 & 0 & \cdots & 0 & 0 & -2 & 2
\end{array}\right)
$$

The corresponding Dynkin diagram is given in figure 1. One recognizes the Dynkin diagram of the overextension $B_{n}^{\wedge \wedge}$ of the finite-dimensional algebra $B_{n}$, which is hyperbolic for $n \leq 8$. For $n=8$, one recovers the Kac-Moody billiard of $B E_{10}$ given in [3], which controls the asymptotic dynamics of the low-energy bosonic sectors of the heterotic and type I superstrings. The hyperbolic character of $B E_{10}$ is another way to see that these models are chaotic.

Note that for $n=3$, one can oxidize further to $D=6$ dimensions [11]. The corresponding theory is gravity + a 2 -form, with no dilaton (there is a self-duality condition on the field strength of the 2-form, which only removes the degeneracy of the 2-form walls and which is not mandatory from the billiard point of view). 


\subsection{The $C_{n}$ sequence}

The oxidation end point is the $D=4$ theory whose Lagrangian is given by

$$
\begin{aligned}
\mathcal{L}_{4}= & R \star \mathbb{1}-\star d \vec{\phi} \wedge d \vec{\phi}-\frac{1}{2} \sum_{\alpha} e^{2 \vec{\sigma}_{\alpha} \cdot \vec{\phi}} \star\left(d \chi^{\alpha}+\cdots\right) \wedge\left(d \chi^{\alpha}+\cdots\right) \\
& -\frac{1}{2} \sum_{a=1}^{n-1} e^{\vec{e}_{a} \cdot \vec{\phi} \sqrt{2}} \star d A_{(1)}^{a} \wedge d A_{(1)}^{a}
\end{aligned}
$$

where the ellipsis complete the "curvatures" of the $\chi$ 's [11. The $(n-1)$ dilatons $\vec{\phi}=\left(\phi^{1}, \ldots, \phi^{n-1}\right)$ are associated with the Cartan subalgebra of $S p(2 n-2, R)$ and the $\frac{1}{2} n(n-1)$ axions $\chi^{\alpha}$ are associated with the positive roots of $\operatorname{Sp}(2 n-2, R)$. The fields $A_{(1)}^{a}$ are one-forms. The $\vec{\sigma}_{\alpha}$ are the positive roots of $S p(2 n-2, R)$; these can be written in terms of an orthonormalized basis of $(n-1)$ vectors in Euclidean space $\left(\vec{e}_{a} \cdot \vec{e}_{b}=\delta_{a b}\right) \vec{e}_{a}(a=1, \cdots, n-1)$ as

$$
\vec{\sigma}_{\alpha}=\left\{\sqrt{2} \vec{e}_{a}, \frac{1}{\sqrt{2}}\left(\vec{e}_{a} \pm \vec{e}_{b}\right), a>b\right\} .
$$

The normalization is such that the long roots have squared length equal to two. The notation $\vec{\sigma}_{\alpha} \cdot \vec{\phi}$ means

$$
\vec{\sigma}_{\alpha} \cdot \vec{\phi} \equiv \sum_{a=1}^{n-1} \sigma_{\alpha}^{a} \phi^{a}
$$

The simple roots are

$$
\sqrt{2} \vec{e}_{1} \quad \text { and } \quad \frac{1}{\sqrt{2}}\left(\vec{e}_{a+1}-\vec{e}_{a}\right), a=1, \ldots, n-2 .
$$

The walls are here given by the curvature wall $2 \beta^{1}$, the symmetry walls

$$
\beta^{3}-\beta^{2} \text { and } \beta^{2}-\beta^{1},
$$

the electric and magnetic walls of the axions

$$
w^{E, \chi^{\alpha}}=\vec{\sigma}_{\alpha} \cdot \vec{\phi} \quad \text { and } \quad w_{i j}^{M, \chi^{\alpha}}=\beta^{i}+\beta^{j}-\vec{\sigma}_{\alpha} \cdot \vec{\phi}
$$

and the electric and magnetic walls of the 1-forms

$$
w_{i}^{E, A^{a}}=\beta^{i}+\frac{\sqrt{2}}{2} \vec{e}_{a} \cdot \vec{\phi} \quad \text { and } \quad w_{i}^{M, A^{a}}=\beta^{i}-\frac{\sqrt{2}}{2} \vec{e}_{a} \cdot \vec{\phi}
$$


The dominant walls are

$$
\begin{aligned}
& w_{-1}=\beta^{3}-\beta^{2} \\
& w_{0}=\beta^{2}-\beta^{1} \\
& w_{1}=\beta^{1}-\frac{\sqrt{2}}{2} \phi^{n-1} \\
& w_{2}=\frac{\sqrt{2}}{2}\left(\phi^{n-1}-\phi^{n-2}\right) \\
& \ldots \\
& w_{n-2}=\frac{\sqrt{2}}{2}\left(\phi^{3}-\phi^{2}\right) \\
& w_{n-1}=\frac{\sqrt{2}}{2}\left(\phi^{2}-\phi^{1}\right) \\
& w_{n}=\sqrt{2} \phi^{1}
\end{aligned}
$$

The Cartan matrix is given by

$$
A=\left(\begin{array}{ccccccccc}
2 & -1 & 0 & 0 & \cdots & 0 & 0 & 0 & 0 \\
-1 & 2 & -1 & 0 & \cdots & 0 & 0 & 0 & 0 \\
0 & -2 & 2 & -1 & \cdots & 0 & 0 & 0 & 0 \\
0 & 0 & -1 & 2 & \cdots & 0 & 0 & 0 & 0 \\
\vdots & \vdots & \vdots & \vdots & \ddots & \vdots & \vdots & \vdots & \vdots \\
0 & 0 & 0 & 0 & \cdots & 2 & -1 & 0 & 0 \\
0 & 0 & 0 & 0 & \cdots & -1 & 2 & -1 & 0 \\
0 & 0 & 0 & 0 & \cdots & 0 & -1 & 2 & -2 \\
0 & 0 & 0 & 0 & \cdots & 0 & 0 & -1 & 2
\end{array}\right)
$$

The Dynkin diagram is given in figure 1. Again we recognize the Dynkin diagram of the overextension $C_{n}^{\wedge \wedge}$. It is hyperbolic for $n \leq 4$.

\subsection{The $D_{n}$ sequence}

The oxidation end point Lagrangian in $D=n+2$ dimensions is the following

$$
\mathcal{L}_{D}=R \star \mathbb{1}-\star d \phi \wedge d \phi-\frac{1}{2} e^{a \sqrt{2} \phi} \star d B \wedge d B
$$

where $B$ is a 2 -form and $a^{2}=8 / n$. 
Besides the curvature and the symmetry walls

$$
\begin{aligned}
& w_{-1}=\beta^{n+1}-\beta^{n}, \quad w_{0}=\beta^{n}-\beta^{n-1} \\
& w_{2}=\beta^{n-1}-\beta^{n-2}, \ldots, w_{n-1}=\beta^{2}-\beta^{1},
\end{aligned}
$$

we get here the 2 -form electric and magnetic walls

$$
\begin{aligned}
& w_{i j}^{E}=\beta^{i}+\beta^{j}+a \frac{\sqrt{2}}{2} \phi \\
& w_{i_{1} \ldots i_{n-2}}^{M}=\beta^{i_{1}}+\ldots+\beta^{i_{n-2}}-a \frac{\sqrt{2}}{2} \phi .
\end{aligned}
$$

The dominant ones are the $w_{-1}, w_{0}, w_{2}, \ldots, w_{n-1}$ defined above and

$$
\begin{aligned}
& w_{n}=\beta^{1}+\beta^{2}+a \frac{\sqrt{2}}{2} \phi \\
& w_{1}=\beta^{1}+\beta^{2}+\ldots+\beta^{n-2}-a \frac{\sqrt{2}}{2} \phi .
\end{aligned}
$$

The Cartan matrix is given by

$$
A=\left(\begin{array}{ccccccccc}
2 & -1 & 0 & 0 & \cdots & 0 & 0 & 0 & 0 \\
-1 & 2 & 0 & -1 & \cdots & 0 & 0 & 0 & 0 \\
0 & 0 & 2 & -1 & \cdots & 0 & 0 & 0 & 0 \\
0 & 0 & -1 & 2 & \cdots & 0 & 0 & 0 & 0 \\
\vdots & \vdots & \vdots & \vdots & \ddots & \vdots & \vdots & \vdots & \vdots \\
0 & 0 & 0 & 0 & \cdots & 2 & -1 & 0 & 0 \\
0 & 0 & 0 & 0 & \cdots & -1 & 2 & -1 & -1 \\
0 & 0 & 0 & 0 & \cdots & 0 & -1 & 2 & 0 \\
0 & 0 & 0 & 0 & \cdots & 0 & -1 & 0 & 2
\end{array}\right)
$$

It is the generalized Cartan matrix of $D_{n}^{\wedge \wedge}=D E_{n+2}$ which is known to be hyperbolic for $n \leq 8$. The corresponding Dynkin diagram is given in figure 1 .

For $n=8$, one gets the last hyperbolic algebra in this family, namely $D E_{10} \equiv D_{8}^{\wedge \wedge}$, [3]. For $n=24$, which is the case relevant to the bosonic string, one gets $D_{24}^{\wedge}$. 


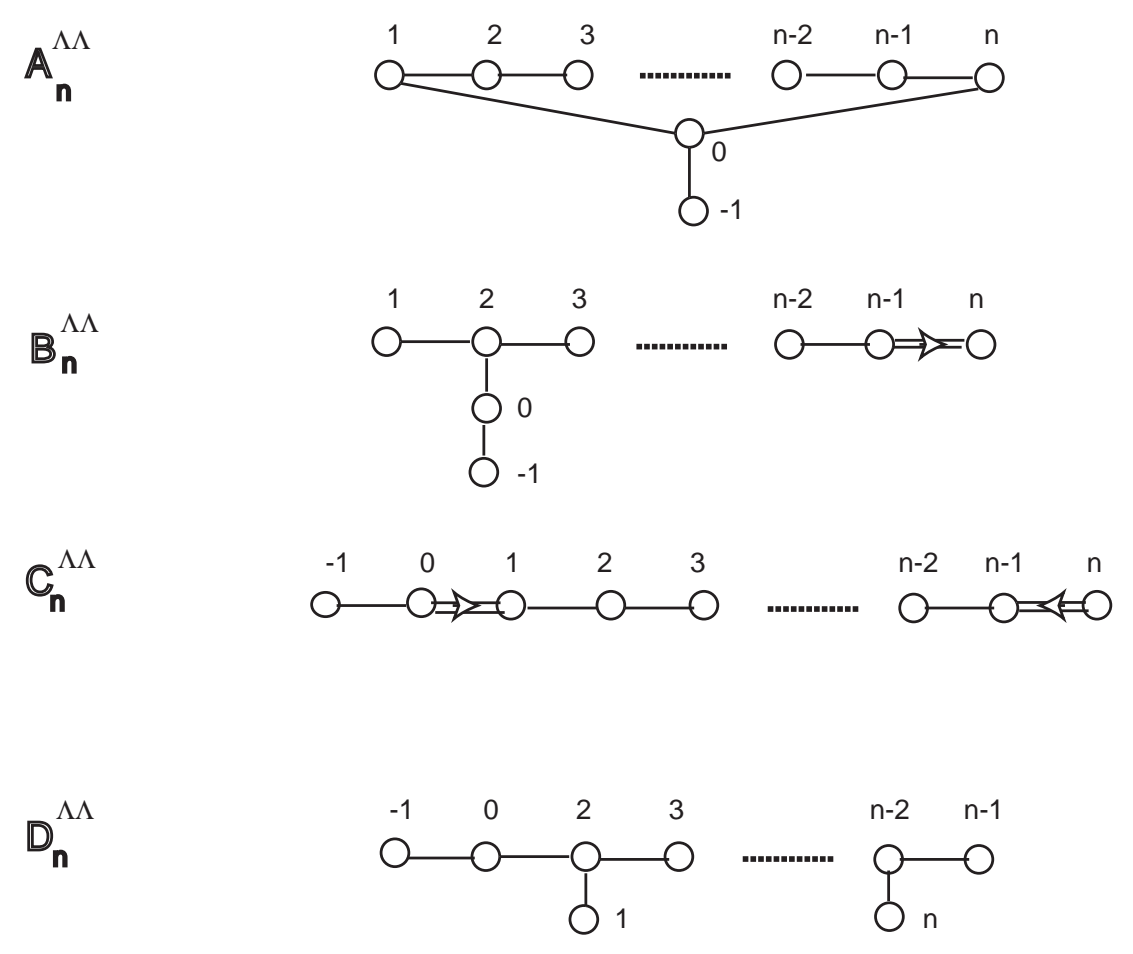

Figure 1.

\section{Models associated with exceptional groups}

\subsection{The $G_{2}$-sequence}

The oxidation end point is the Einstein-Maxwell system in $D=5$ with an extra FFA term [11]:

$$
\mathcal{L}_{5}=R \star \mathbb{1}-\frac{1}{2} \star F \wedge F+\frac{1}{3 \sqrt{3}} F \wedge F \wedge A, \quad F=d A .
$$

Besides the curvature and the symmetry walls, there are one-form electric walls $w_{i}^{E}=\beta^{i}$ and magnetic walls $w_{i j k}^{M}=\beta^{i}+\beta^{j}+\beta^{k}, \quad i<j<k$. The dominant walls are

$$
\begin{aligned}
& w_{-1}=\beta^{4}-\beta^{3} \\
& w_{0}=\beta^{3}-\beta^{2}
\end{aligned}
$$




$$
\begin{aligned}
& w_{1}=\beta^{2}-\beta^{1} \\
& w_{2}=\beta^{1} .
\end{aligned}
$$

The Cartan matrix reads

$$
A=\left(\begin{array}{cccc}
2 & -1 & 0 & 0 \\
-1 & 2 & -1 & 0 \\
0 & -1 & 2 & -1 \\
0 & 0 & -3 & 2
\end{array}\right)
$$

which is the generalized Cartan matrix of $G_{2}^{\wedge \wedge}$. Its Dynkin diagram is given in figure 2; the algebra is hyperbolic [8]. See also [18] for the relevance of $G_{2}^{\wedge \wedge}$ to this system.

\subsection{The $F_{4}$-sequence}

The oxidation end point of the $F_{4}$ sequence is a $D=6$ dimensional theory containing the metric, a dilaton $(\phi)$, an axion $(\chi)$, two one-forms $\left(A^{ \pm}\right)$, a two-form $(B)$ and a self-dual 3-form field strength $(G)$ [11]. The Lagrangian is given by

$$
\begin{aligned}
\mathcal{L}_{6}= & R \star \mathbb{1}-\star d \phi \wedge d \phi-\frac{1}{2} e^{2 \phi} \star d \chi \wedge d \chi-\frac{1}{2} e^{-2 \phi} \star H \wedge H \\
& -\frac{1}{2} \star G \wedge G-\frac{1}{2} e^{\phi} \star F^{+} \wedge F^{+}-\frac{1}{2} e^{-\phi} \star F^{-} \wedge F^{-} \\
& -\frac{1}{\sqrt{2}} \chi H \wedge G-\frac{1}{2} A^{+} \wedge F^{+} \wedge H-\frac{1}{2} A^{+} \wedge F^{-} \wedge G .
\end{aligned}
$$

The field strengths are given in terms of potentials as follows:

$$
\begin{aligned}
& F^{+}=d A^{+}+\frac{1}{\sqrt{2}} \chi d A^{-} \\
& F^{-}=d A^{-} \\
& H=d B+\frac{1}{2} A^{-} \wedge d A^{-} \\
& G=d C-\frac{1}{\sqrt{2}} \chi H-\frac{1}{2} A^{+} \wedge d A^{-} .
\end{aligned}
$$

Besides the curvature and the symmetry walls we get here 
1. Electric walls

$$
\begin{aligned}
& w^{E, \chi}=\phi \\
& w_{i}^{E, A^{+}}=\beta^{i}+\frac{\phi}{2} \\
& w_{i}^{E, A^{-}}=\beta^{i}-\frac{\phi}{2} \\
& w_{i j}^{E, B}=\beta^{i}+\beta^{j}-\phi, \quad i<j \\
& w_{i j}^{E, C}=\beta^{i}+\beta^{j}, \quad i<j
\end{aligned}
$$

2. Magnetic walls

$$
\begin{aligned}
& w_{i j k \ell}^{M, \chi}=\beta^{i}+\beta^{j}+\beta^{k}+\beta^{\ell}-\phi, \quad i<j<k<\ell \\
& w_{i j k}^{M, A^{+}}=\beta^{i}+\beta^{j}+\beta^{k}-\frac{\phi}{2}, \quad i<j<k \\
& w_{i j k}^{M, A^{-}}=\beta^{i}+\beta^{j}+\beta^{k}+\frac{\phi}{2}, \quad i<j<k \\
& w_{i}^{M, B}=\beta^{i}+\phi \\
& w_{i}^{M, C}=\beta^{i}
\end{aligned}
$$

The dominant walls are

$$
\begin{aligned}
& w_{-1}=\beta^{5}-\beta^{4} \\
& w_{0}=\beta^{4}-\beta^{3} \\
& w_{1}=\beta^{3}-\beta^{2} \\
& w_{2}=\beta^{2}-\beta^{1} \\
& w_{3}=\beta^{1}-\frac{\phi}{2} \\
& w_{4}=\phi .
\end{aligned}
$$

The Cartan matrix is given by

$$
A=\left(\begin{array}{cccccc}
2 & -1 & 0 & 0 & 0 & 0 \\
-1 & 2 & -1 & 0 & 0 & 0 \\
0 & -1 & 2 & -1 & 0 & 0 \\
0 & 0 & -1 & 2 & -1 & 0 \\
0 & 0 & 0 & -2 & 2 & -1 \\
0 & 0 & 0 & 0 & -1 & 2
\end{array}\right)
$$

This is the generalized Cartan matrix of $F_{4}^{\wedge \wedge}$; its Dynkin diagram is given in figure 2. The overextended algebra is hyperbolic. 


\subsection{The $E_{6}$-sequence}

The oxidation end point of the $E_{6}$ sequence is $D=8$ and the associated theory is the smallest obtainable as a truncation of maximal supergravity in which the 3 -form potential is retained. It comprises the metric, a dilaton and an axion, $\chi$, together with the 3-form, $C$ [11]. The 8-dimensional Lagrangian is given by

$$
\mathcal{L}_{8}=R \star \mathbb{1}-\star d \phi \wedge d \phi-\frac{1}{2} e^{2 \sqrt{2} \phi} \star d \chi \wedge d \chi-\frac{1}{2} e^{-\sqrt{2} \phi} \star G \wedge G+\chi G \wedge G,
$$

where $G=d C$. In addition to the gravitational and symmetry walls, there are the electric and magnetic walls of the scalar

$$
w^{E, \chi}=\sqrt{2} \phi \quad \text { and } \quad w_{i_{1} \ldots i_{6}}^{M, \chi}=\beta^{i_{1}}+\ldots+\beta^{i_{6}}+\sqrt{2} \phi
$$

and those coming from the 3-form

$$
\begin{aligned}
& w_{i j k}^{E, C}=\beta^{i}+\beta^{j}+\beta^{k}-\frac{\sqrt{2}}{2} \phi \\
& w_{i j k}^{M, C}=\beta^{i}+\beta^{j}+\beta^{k}+\frac{\sqrt{2}}{2} \phi .
\end{aligned}
$$

The dominant walls are the symmetry walls

$$
\begin{aligned}
& w_{-1}=\beta^{7}-\beta^{6}, \quad w_{0}=\beta^{6}-\beta^{5} \\
& w_{1}=\beta^{2}-\beta^{1}, \quad w_{2}=\beta^{3}-\beta^{2}, \quad w_{3}=\beta^{4}-\beta^{3}, \quad w_{6}=\beta^{5}-\beta^{4}
\end{aligned}
$$

and

$$
\begin{aligned}
& w_{4}=\beta^{1}+\beta^{2}+\beta^{3}-\frac{\sqrt{2}}{2} \phi \\
& w_{5}=\sqrt{2} \phi .
\end{aligned}
$$

The Cartan matrix is given by

$$
A=\left(\begin{array}{cccccccc}
2 & -1 & 0 & 0 & 0 & 0 & 0 & 0 \\
-1 & 2 & 0 & 0 & 0 & 0 & 0 & -1 \\
0 & 0 & 2 & -1 & 0 & 0 & 0 & 0 \\
0 & 0 & -1 & 2 & -1 & 0 & 0 & 0 \\
0 & 0 & 0 & -1 & 2 & -1 & 0 & -1 \\
0 & 0 & 0 & 0 & -1 & 2 & -1 & 0 \\
0 & 0 & 0 & 0 & 0 & -1 & 2 & 0 \\
0 & -1 & 0 & 0 & -1 & 0 & 0 & 2
\end{array}\right)
$$


It is the generalized Cartan matrix of the hyperbolic algebra $E_{6}^{\wedge \wedge}$. The Dynkin diagram is given in figure 2.

\subsection{The $E_{7}$-sequence}

This sequence is obtained as a consistent (albeit non supersymmetric) truncation of $D=9$ maximal supergravity to the theory whose bosonic sector comprises the metric, a dilaton, a 1-form, $A$, and a 3 -form potential $C$ [11]. The Lagrangian reads as

$$
\begin{aligned}
\mathcal{L}_{9}= & R \star \mathbb{1}-\star d \phi \wedge d \phi-\frac{1}{2} e^{\frac{2 \sqrt{2}}{\sqrt{7}} \phi} \star d C \wedge d C \\
& -\frac{1}{2} e^{-\frac{4 \sqrt{2}}{\sqrt{7}} \phi} \star d A \wedge d A-\frac{1}{2} d C \wedge d C \wedge A .
\end{aligned}
$$

Besides the curvature and the symmetry walls, we have here the electric and magnetic malls of the 1 -form

$$
\begin{aligned}
& w_{i}^{E, A}=\beta^{i}-\frac{2 \sqrt{2}}{\sqrt{7}} \phi \\
& w_{i_{1} \ldots i_{6}}^{M, A}=\beta^{i_{1}}+\ldots \beta^{i_{6}}+\frac{2 \sqrt{2}}{\sqrt{7}} \phi
\end{aligned}
$$

and the corresponding walls of the 3 -form

$$
\begin{aligned}
& w_{i j k}^{E, C}=\beta^{i}+\beta^{j}+\beta^{k}+\frac{\sqrt{2}}{\sqrt{7}} \phi \\
& w_{i j k \ell}^{M, C}=\beta^{i}+\beta^{j}+\beta^{k}+\beta^{\ell}-\frac{\sqrt{2}}{\sqrt{7}} \phi
\end{aligned}
$$

The dominant walls are the symmetry walls

$$
w_{-1}=\beta^{8}-\beta^{7}, w_{0}=\beta^{7}-\beta^{6}, w_{1}=\beta^{6}-\beta^{5}, \ldots, w_{5}=\beta^{2}-\beta^{1}
$$

and

$$
\begin{aligned}
& w_{6}=\beta^{1}-\frac{2 \sqrt{2}}{\sqrt{7}} \phi \\
& w_{7}=\beta^{1}+\beta^{2}+\beta^{3}+\frac{\sqrt{2}}{\sqrt{7}} \phi .
\end{aligned}
$$


The Cartan matrix reads

$$
A=\left(\begin{array}{ccccccccc}
2 & -1 & 0 & 0 & 0 & 0 & 0 & 0 & 0 \\
-1 & 2 & -1 & 0 & 0 & 0 & 0 & 0 & 0 \\
0 & -1 & 2 & -1 & 0 & 0 & 0 & 0 & 0 \\
0 & 0 & -1 & 2 & -1 & 0 & 0 & 0 & 0 \\
0 & 0 & 0 & -1 & 2 & -1 & 0 & 0 & -1 \\
0 & 0 & 0 & 0 & -1 & 2 & -1 & 0 & 0 \\
0 & 0 & 0 & 0 & 0 & -1 & 2 & -1 & 0 \\
0 & 0 & 0 & 0 & 0 & 0 & -1 & 2 & 0 \\
0 & 0 & 0 & 0 & -1 & 0 & 0 & 0 & 2
\end{array}\right)
$$

The Dynkin diagram is given in figure 2; again it is the overextension $E_{7}^{\wedge \wedge}$, which is hyperbolic.

In fact, one can view the above Lagrangian as the reduction to 9 spacetime dimensions of the type IIB supergravity theory in which one keeps only the metric and the chiral 4-form [11]. From the point of view of getting the correct walls, the self-duality condition on the field strength is actually not necessary: without the chirality condition, each 4 -form walls would simply appear twice (once as electric, once as magnetic wall).

\subsection{The $E_{8}$-sequence}

The oxidation end point is $D=11$-dimensional supergravity whose bosonic sector is given by

$$
\mathcal{L}_{11}=R \star \mathbb{1}-\frac{1}{2} \star d C \wedge d C-\frac{1}{6} d C \wedge d C \wedge C
$$

$C$ is a 3 -form.

Besides the curvature and the symmetry walls, we have here the electric and magnetic walls of the 3 -form which read as

$$
\begin{aligned}
& w_{i j k}^{E}=\beta^{i}+\beta^{j}+\beta^{k} \\
& w_{i_{1} \ldots i_{6}}^{M}=\beta^{i_{1}}+\ldots+\beta^{i_{6}} .
\end{aligned}
$$

The dominant walls are the symmetry walls

$$
w_{-1}=\beta^{10}-\beta^{9}, w_{0}=\beta^{9}-\beta^{8}, w_{1}=\beta^{8}-\beta^{7}, \ldots, w_{7}=\beta^{2}-\beta^{1}
$$


and

$$
w_{8}=\beta^{1}+\beta^{2}+\beta^{3} .
$$

The Cartan matrix is

$$
A=\left(\begin{array}{cccccccccc}
2 & -1 & 0 & 0 & 0 & 0 & 0 & 0 & 0 & 0 \\
-1 & 2 & -1 & 0 & 0 & 0 & 0 & 0 & 0 & 0 \\
0 & -1 & 2 & -1 & 0 & 0 & 0 & 0 & 0 & 0 \\
0 & 0 & -1 & 2 & -1 & 0 & 0 & 0 & 0 & 0 \\
0 & 0 & 0 & -1 & 2 & -1 & 0 & 0 & -1 & 0 \\
0 & 0 & 0 & 0 & -1 & 2 & -1 & 0 & 0 & 0 \\
0 & 0 & 0 & 0 & 0 & -1 & 2 & 0 & 0 & -1 \\
0 & 0 & 0 & 0 & 0 & 0 & -1 & 2 & -1 & 0 \\
0 & 0 & 0 & 0 & 0 & 0 & 0 & -1 & 2 & 0 \\
0 & 0 & 0 & 0 & 0 & 0 & -1 & 0 & 0 & 2
\end{array}\right)
$$

As pointed out in [3] this is the Cartan matrix of the overextension $E_{8}^{\wedge \wedge}$, better known as $E_{10}$. As shown in that paper, it is also the Cartan matrix relevant to type IIA supergravity in ten dimensions (dimensional reduction) as well as type IIB. In fact, massive type IIA supergravity in ten dimensions [19 is also described by the same billiard, as one can easily verify by using the formulation of [20]: the new wall associated with the mass term can be expressed as a linear combination with positive (integer) coefficients of the dominant walls and is thus subdominant (note that it has squared norm equal to 2).

The Dynkin diagram is given in figure 2; it is hyperbolic. The relevance of $E_{10}$ in the supergravity context was first conjectured in [21. 


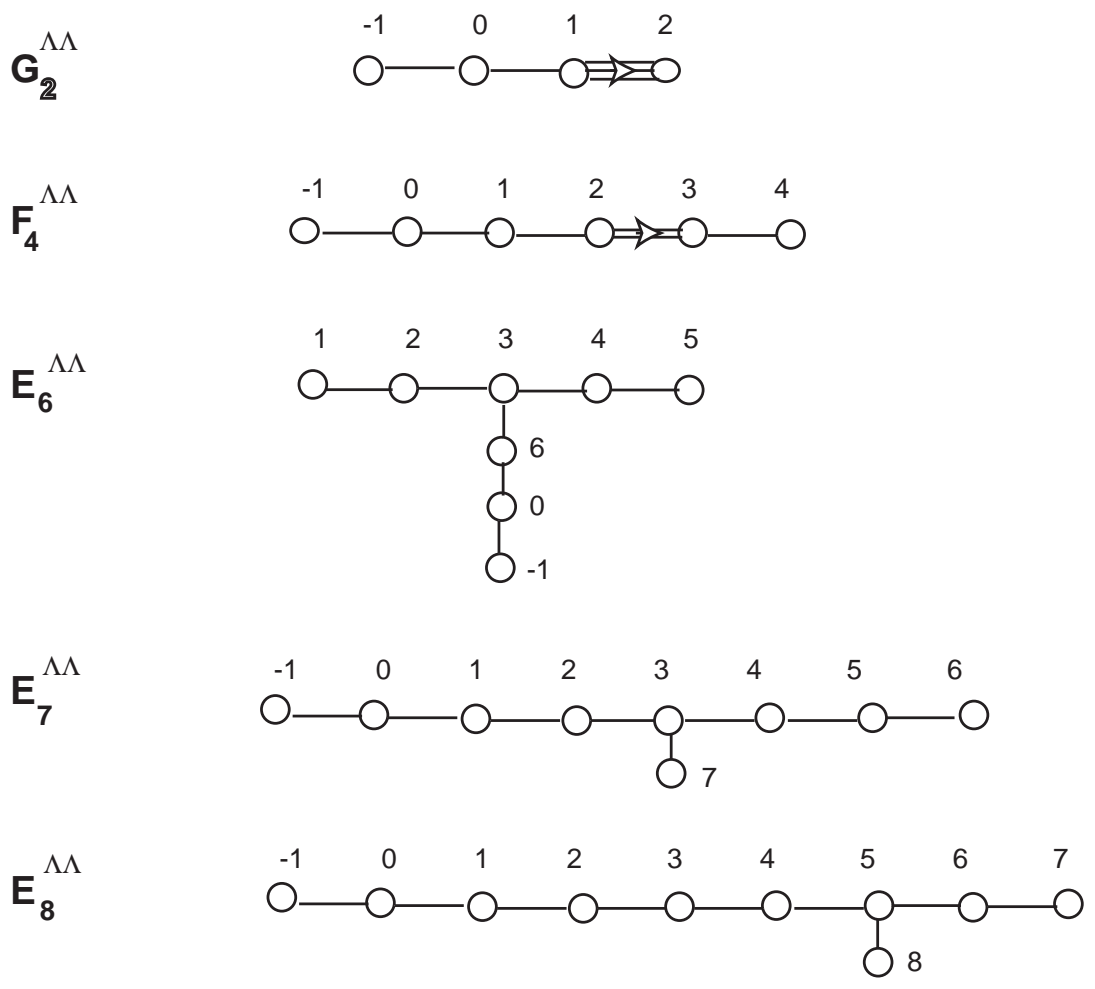

Figure 2

\section{Conclusions}

In this paper, we have provided models that realize the canonical Lorentzian extensions, or overextensions, of all the finite-dimensional Lie algebras, in the sense that their asymptotic dynamics in the vicinity of a spacelike singularity is a billiard motion in the fundamental Weyl chamber of the corresponding Kac-Moody algebras. We have also shown that the computation can be done at any point along the oxidation sequence.

The explicit proof given above of the appearance of the overextensions was achieved by a case by case analysis, and was based on the calculation of the Cartan matrix of the billiard as it appears in the highest possible dimension (i.e. at the upper edge of the oxidation sequence). As we said, another natural dimension in which to compute the Cartan matrix of the billiard is the lower edge of the oxidation sequence, namely in $D=3$ spacetime dimen- 
sions, where the scalar Lagrangian is the one of a $G / H$ coset model. In this concluding section, we complete our work by indicating how the calculation goes in $D=3$. We are going to see that the calculation in this dimension has the advantage of streamlining the essential structural elements which are at the origin of the appearance of overextensions. This allows one to better understand why the overextension of $\mathcal{G}$ systematically appears.

In $D=3$ dimensions, each Lagrangian model of [10, 11] exhibits the following general structure

$$
\mathcal{L}_{3}=R \star \mathbb{1}-\sum_{\alpha=1}^{r} \star d \phi^{\alpha} \wedge d \phi^{\alpha}-\frac{1}{2} \sum_{A} e^{2 \vec{\sigma}_{A} \cdot \vec{\phi}} \star\left(d \chi^{A}+\cdots\right) \wedge\left(d \chi^{A}+\cdots\right)
$$

where the dilatons $\phi^{\alpha}(\alpha=1, \ldots, r)$ are associated with a Cartan subalgebra of the simple Lie algebra $\mathcal{G}$ of rank $r$ and the scalar axions $\chi^{A}$ are associated with all the positive roots $\vec{\sigma}_{A}$ of $\mathcal{G}$. In this Lagrangian, the ellipsis $\cdots$ result from modifications of the "curvatures". Such modifications are essential for ensuring the presence of an exact $G$-symmetry. However, they are not important for computing the Cartan matrix of the corresponding cosmological billiard. The notation $\vec{\sigma}_{A} \cdot \vec{\phi}$ stands for

$$
\vec{\sigma}_{A} \cdot \vec{\phi} \equiv \sum_{\alpha=1}^{r} \sigma_{A}^{\alpha} \phi^{\alpha}
$$

It could also be denoted $\sigma_{A}(\phi)$ to emphasize that $\sigma_{A}$ is a linear form of the $\phi$ 's. A key feature for deriving our result is the fact that the norms of the roots in the Lagrangians of 11] are such that the long roots (and in particular the highest root $\theta$ ) have squared length equal to 2 . Indeed, if we were to focus only on the $G$-symmetry in $D=3$ dimensions, one could multiply the scalar part of the Lagrangian (7.1) by an arbitrary number $k \neq 0$ without changing its $G$-invariance. This would multiply the norms of all the nongravitational roots by $1 / k$. However, such models in which one changes the relative normalization of the Einstein-Hilbert action with respect to the scalar coset action cannot be oxidized to dimensions $D>3$ [11]. Our computation below shows that the correct relative normalization for "oxidability" to higher dimensions is also crucial for getting the overextension.

Note that, in $D=3$ all the non-gravitational walls are due to the energy associated to scalar fields: the axions $\chi^{A}$. (We recall that, in $D=3$, one can use dualisations to replace the other fields, i.e. the one-forms if any, by scalars). Still, we must take into account both the electric walls of the axions 
(linked to the time derivatives of $\chi^{A}$ ) and their magnetic walls (linked to their space derivatives). The axion electric walls depend only on the dilatons and read

$$
w^{E, \chi^{A}}=\vec{\sigma}_{A} \cdot \vec{\phi}
$$

The axion magnetic walls are

$$
w_{i}^{M, \chi^{A}}=\beta^{i}-\vec{\sigma}_{A} \cdot \vec{\phi}
$$

Finally, since $d=2$, there is only one symmetry wall

$$
w^{S}=\beta^{2}-\beta^{1} .
$$

Among these, the dominant walls are

1. The electric walls built from a set of simple roots $\vec{\sigma}_{\alpha}(\alpha=1, \cdots, r)$ and written as

$$
w_{\alpha}=\vec{\sigma}_{\alpha} \cdot \vec{\phi}
$$

Indeed, all the other electric walls will be, by definition, combinations of such simple walls with positive coefficients.

2. The magnetic wall associated (in view of $(7.3)$ ) to the lowest $\beta^{i}$ (i.e. $\beta^{1}$, because $\beta^{2}=\beta^{1}+w^{S}$ ), and to the highest root of $\mathcal{G}$. This yields as dominant magnetic wall

$$
w_{0}=\beta^{1}-\vec{\theta} \cdot \vec{\phi}
$$

where $\vec{\theta}=\sum_{\alpha} n_{\alpha} \vec{\sigma}_{\alpha}$ denotes the highest root of $\mathcal{G}$.

3. The symmetry wall:

$$
w_{-1}=w^{S}=\beta^{2}-\beta^{1} .
$$

Let us now notice that the two linear forms $-\beta^{1}$ and $\beta^{2}$ have zero norm and scalar product equal to +1 . Moreover, they are, by definition orthogonal (in the sense of the co-metric (2.3) ) to all the $\phi$-dependent forms. Therefore they play exactly the rôle of the additional null roots $u_{1}$ and $u_{2}$ introduced in section 4 , and can be identified with these. Explicitly: $-\beta^{1} \rightarrow u_{2}$ and $\beta^{2} \rightarrow u_{1}$. One then sees that the dominant wall forms above can be identified with the simple roots of the overextension $\mathcal{G}^{\wedge}$ of $\mathcal{G}$. More precisely, the 
relevant electric wall-forms are the simple roots of $\mathcal{G}$, the magnetic wallform is the root which does the affine extension $\mathcal{G}^{\wedge}$ and the symmetry wallform is the one responsible for the overextension. This completes our second (general) proof of the appearance of the overextension $\mathcal{G}^{\wedge \wedge}$ in the cosmological billiards.

The magnetic wall (7.6) is in fact also a symmetry wall in the last dimension of the oxidation sequence; thus, as all gravitational walls, it has squared norm equal to two. Since $-\beta^{1}$ has zero norm, this explains the origin of the normalization of the highest root $\vec{\theta}$ in our context.

Finally, we note that the invariance of the billiard under dimensional reduction may help in conjecturing which symmetry group arises in the toroidal compactification down to three dimensions of a theory given in $D>3$ dimensions, without having to actually carry out this reduction.

\section{Acknowledgements}

M.H. is grateful to Bernard Julia for informative discussions. The work of S.d.B., M.H. and C.S. is supported in part by the "Actions de Recherche Concertées" of the "Direction de la Recherche Scientifique - Communauté Française de Belgique", by a "Pôle d'Attraction Interuniversitaire" (Belgium), by IISN-Belgium (convention 4.4505.86), by Proyectos FONDECYT 1970151 and 7960001 (Chile) and by the European Commission RTN programme HPRN-CT-00131, in which they are associated to K. U. Leuven.

\section{References}

[1] V.A. Belinskii, I.M. Khalatnikov and E.M. Lifshitz, "Oscillatory approach to a singular point in the relativistic cosmology," Adv. Phys. 19, 525 (1970); "A general solution of the Einstein equations with a time singularity," Adv. Phys. 31, 639 (1982).

[2] T. Damour and M. Henneaux, "Chaos in superstring cosmology," Phys. Rev. Lett. 85, 920 (2000) arXiv:hep-th/0003139]; [See also short version in Gen. Rel. Grav. 32, 2339 (2000).] 
[3] T. Damour and M. Henneaux, "E(10), BE(10) and arithmetical chaos in superstring cosmology," Phys. Rev. Lett. 86, 4749 (2001) arXiv:hepth/0012172].

[4] D.M. Chitre, Ph. D. thesis, University of Maryland, 1972.

[5] C.W. Misner, in: D. Hobill et al. (Eds), Deterministic chaos in general relativity, Plenum, 1994, pp. 317-328 gr-qc/9405068.

[6] B. S. Dewitt, "Quantum Theory Of Gravity. 1. The Canonical Theory," Phys. Rev. 160, 1113 (1967).

[7] T. Damour and M. Henneaux and H. Nicolai, in preparation.

[8] T. Damour, M. Henneaux, B. Julia and H. Nicolai, "Hyperbolic KacMoody algebras and chaos in Kaluza-Klein models," Phys. Lett. B 509, 323 (2001) arXiv:hep-th/0103094.

[9] J. Demaret, M. Henneaux and P. Spindel, "Nonoscillatory Behavior In Vacuum Kaluza-Klein Cosmologies," Phys. Lett. 164B, 27 (1985).

[10] P. Breitenlohner, D. Maison and G. W. Gibbons, "Four-Dimensional Black Holes From Kaluza-Klein Theories," Commun. Math. Phys. 120, 295 (1988).

[11] E. Cremmer, B. Julia, H. Lu and C. N. Pope, arXiv:hep-th/9909099.

[12] T. Damour and M. Henneaux, "Oscillatory behaviour in homogeneous string cosmology models," Phys. Lett. B 488, 108 (2000) arXiv:hepth/0006171.

[13] B. Julia, in Lectures in Applied Mathematics, AMS-SIAM, vol 21 (1985), p.355.

[14] G. A. Margulis, "Applications of ergodic theory to the investigation of manifolds of negative curvature," Funct. Anal. Appl. 4, 335 (1969); R. E. Howe and C. C. Moore, "Asymptotic properties of unitary representations," J. Functional Analysis 32, $72-96$ (1979); R. Zimmer, Ergodic Theory and Semisimple Groups, (Birkhauser, Boston, 1984); A. Eskin and C. McMullen, "Mixing, counting and equidistribution in Lie groups", Duke Math. J. 71, 181-209 (1993). 
[15] P. Goddard and D. I. Olive, "Algebras, Lattices And Strings," DAMTP83/22 Talks given at the Workshop on Vertex Operators in Mathematics and Physics, Berkeley, Calif..

[16] V. G. Kac, Infinite Dimensional Lie Algebras, third edition (Cambridge University Press, 1990).

[17] E. B. Vinberg, "Hyperbolic reflection groups", Russian Math. Surveys 40, 31-75 (1985); E. B. Vinberg, ed. , Geometry II, Encyclopedia of Mathematical Sciences, Vol. 29 (Springer-Verlag, Berlin, 1993).

[18] S. Mizoguchi and N. Ohta, Phys. Lett. B 441, 123 (1998) hepth/9807111]; G. Schröder and S. Mizoguchi, Class. Quantum Grav. 17 (2000) 835 [hep-th/9909150].

[19] L. J. Romans, "Massive N=2A Supergravity In Ten-Dimensions," Phys. Lett. B 169, 374 (1986).

[20] E. Bergshoeff, M. de Roo, M. B. Green, G. Papadopoulos and P. K. Townsend, "Duality of Type II 7-branes and 8-branes," Nucl. Phys. B 470, 113 (1996) arXiv:hep-th/9601150.

[21] B. Julia, LPTENS 80/16, Invited paper presented at Nuffield Gravity Workshop, Cambridge, Eng., Jun 22 - Jul 12, 1980. 